\title{
Secure Computation with Information Leaking to an Adversary
}

\author{
[Extended Abstract]
}

\author{
Miklós Ajtai \\ IBM Almaden Research Center \\ 650 Harry Road \\ San Jose, CA 95120 \\ ajtai@almaden.ibm.com
}

\begin{abstract}
Assume that Alice is running a program $P$ on a RAM, and an adversary Bob would like to get some information about the input or output of the program. At each time, during the execution of $P$, Bob is able to see the addresses of the memory cells involved in the instruction which is executed and the name of the instruction. In addition to this, at certain times, Bob can even see the contents of all of the memory cells involved in the instruction. We will call a time when this happens a compromised time. Bob can choose the compromised times in an adaptive way, that is, immediately before the instruction at time $t$ is executed, Bob, using all of the information at his disposal, can decide whether time $t$ will be compromised or not. The only restriction on his choice is, that among $m$ consecutive instructions there can be at most $\varepsilon m$ whose time is compromised, where $\varepsilon>0$ is a small constant. We show that if $m=c\lfloor\log n\rfloor$, where $c>0$ is a large constant, then for each program $P$, using $n$ memory cells and time $T=O(\operatorname{poly}(n))$, Alice can construct a functionally equivalent program $P^{\prime}$, such that the probability that Bob gets any nontrivial information about the input of $P$ is negligible, and the time and space requirements of $P^{\prime}$ grows, compared to $P$, only by a factor of poly $(\log n)$. We assume that the program $P^{\prime}$ gets its input in an encoded form, namely each input bit $b$ is encoded by a random 0,1 sequence of length $m$ whose parity is $b$. The output bits must be encoded by $P^{\prime}$ in a similar way.

As part of the proof of the result described above we also construct for all positive integers $m$, and for all boolean circuits $C$ of size $n$ a functionally equivalent circuit $C^{\prime}$ of size $O(n \operatorname{poly}(m))$ with the following properties. Assume that an adversary can observe each bit going through the wires of the circuit $C^{\prime}$ independently with a probability of $\varepsilon$, where $\varepsilon>0$ is a small constant, and each input/output bit of $C$ is encoded by $m$ input/output bits of $C^{\prime}$ the same way as described above for RAMs. Then, such an adversary, while observing $C^{\prime}$, can get any information about the input/output
\end{abstract}

Permission to make digital or hard copies of all or part of this work for personal or classroom use is granted without fee provided that copies are not made or distributed for profit or commercial advantage and that copies bear this notice and the full citation on the first page. To copy otherwise, to republish, to post on servers or to redistribute to lists, requires prior specific permission and/or a fee.

STOC'11, June 6-8, 2011, San Jose, California, USA

Copyright 2011 ACM 978-1-4503-0691-1/11/06 ...\$10.00. of the circuit $C$ only with a probability of $n e^{-c m}$, where $c>0$ is a constant.

\section{Categories and Subject Descriptors}

F.1.3 [Theory of Computation]: Computation by Abstract Devices-complexity measures and classes; F.2.3 [Theory of Computation]: Analysis of Algorithms and Problem Complexity_tradeoffs among complexity measures

\section{General Terms}

Theory

\section{INTRODUCTION}

\subsection{The history of the problem.}

The problem of secure computation in the presence of an adversary who has some partial information about the computation was studied for the special case of oblivious computing. In this case the adversary knows the memory access pattern. E.g., for a RAM this means that the adversary knows at each time which memory cells were accessed by the machine at that time. The problem of doing computation relatively efficiently so that such an adversary does not gain any essentially new information was solved first for Turing machines by Pippenger and Fischer [13]. The question for RAMS was formulated by Goldreich in [6], and solutions gradually making the oblivious simulation more efficient and the computational model more realistic were given by Goldreich [6], Ostrovsky [11], [12], Goldreich and Ostrovsky [7], and Ajtai [3], [2]. It is proved in [3] that a program $P$ running on a RAM can be obliviously simulated, with a negligible probability of failure, on a RAM with a random number generator so that the loss of efficiency in terms of time and memory requirements is only a factor of $\log n$, where $n$ is the memory requirement of $P$. (Independently, another solution was given by Damgård, Meldgaard, and Nielsen for the same problem in [4], where the failure probability is 0 .) The present result can be considered as an extension of the result in [3] in the sense that we show that the theorem remains true for a much stronger adversary. From a technical point of view, however, the proof of the present result is completely different from the proof of the results of [3] or the other proofs concerning oblivious simulation. 
The general question of computation performed on a device $D$, in the presence of an adversary who may get some partial information about the inner working of $D$, got much attention in the last decade. The main motivating force was that various side channel attacks, when the adversary through physical measurements may get some information about what is happening in the device, were realized in practice. Ishai, Sahai, and Wagner considered the case in [9], when the device $D$ is a circuit $C$ and the adversary may find out the values of the bits flowing through certain wires of $C$. We will call these the compromised wires. Depending on how the compromised wires are selected by the adversary: adaptively, at random etc., and what can be the total number of compromised wires, there are many possibilities. In [9] rigorous foundations of formulating these type of questions are given. Two types of circuits are considered stateless and stateful circuits. The boolean circuits in the usual sense, where we are interested in hiding the input and output form the adversary and after each evaluation of the circuit no information remains in its wires or gates are the stateless circuits. In this paper we are interested only in stateless circuits so we will refer to them simply as circuits.

The notion of a private circuit is introduced in [9] in the following sense. A circuit is given, and an adversary can access the information in certain wires of the circuit. There may be some restrictions on the choices of the compromised wires. The circuit is private (with respect to an adversary) if the adversary cannot get any information about the input and the output of the circuit. One of the results of [9] is about the case when the adversary chooses adaptively the compromised wires but there is a limit $t$ on the total number of such wires. It is proved in [9], using a technique from the theory of multiparty computations, that for each circuit $C$ of size $n$, there exists an efficiently constructed and functionally equivalent circuit $C^{\prime}$ so that $C^{\prime}$ is private against an adversary, who can choose adaptively $t$ compromised wires, and the size of $C^{\prime}$ is at most $O\left(n t^{2}\right)$. Each input bit and output bit $b$ of $C$ is encoded by a 0,1 -sequence of length $t+1$ whose parity is $b$. Each gate of $C$ is simulated in $C^{\prime}$ by a gadget of size $O\left(t^{2}\right)$. The privacy of the circuit is perfect in the sense that the distribution of the bits seen by the adversary does not depend on the input and output of the circuit. The result remains valid if we allow $t$ compromised wires form each gadget.

In the present paper we prove a theorem which can be formulated in the framework of [9]. Assume that we have and adversary of the following type. The compromised wires are chosen independently, each with probability $p$, and the adversary knows the bits flowing through the compromised wires. Such an adversary will be called a $p$-random adversary. In this paper we show that if $p=\varepsilon$, where $\varepsilon>0$ is a small constant, then for each boolean circuit $C$ of size $n$, we can construct a functionally equivalent circuit $C^{\prime}$, so that $|C|=O\left((\log n)^{4} n\right)$, each input/output bit of $C$ is encoded by the parity of a 0,1 -sequence of length $m=O(\log n)$, and $C$ is private against an $\varepsilon$-random adversary in a statistical sense, that is the probability that any information about the input/output of $C$ will reach the adversary is negligible. In [9] the general case of randomly chosen compromised wires is reduced to the to the worst-case problem using the fact that with high probability the number of compromised wires can be only a constant times larger than its expected value. The results in [9] imply that a private circuit against a $p$ - random adversary can be constructed if $p=O\left((\log n)^{-1}\right)$. Our result improves the probability allowed for the choice of the compromised wires from $p=O\left((\log n)^{-1}\right)$ to a small constant. The construction in [9] however is more efficient if we consider the size of the circuit $C^{\prime}$. The paper [9] contains another construction as well for a private circuit against $p$ random adversaries.

For constructions of private circuits several reasonable additional assumptions can be made, for example Goldwasser and Rothblum in [8] consider circuits with simple leak-proof hardware component. Faust et al. in [5] consider adversaries who does not get individual bits flowing in the device, but some function of them. A general model for handling sidechannel attacks is given by Micali and Reyzin in [10]. They formulate the principle that "computation and only computation leaks information". That is, an inactive part of the memory is not in danger. In our results about computations on RAMs, we follow this principle; only the contents and addresses of those memory cells which are involved in an instruction executed at time $t$, may leak to the adversary at time $t$.

\subsection{Informal description of the results}

We prove two related theorems: a theorem about RAMs, resilient against certain type of attacks and another theorem about constructing private circuits. The circuit theorem is used as a step in the proof about RAMs.

Leak-proof simulation on RAMs. Assume that an adversary tries to get some information about the input/output of a program $P$ running on a RAM with $n$ memory cells each containing $O(\log n)$ bits. At each time, during the execution of $P$, the adversary is able to see the addresses of the memory cells involved in the instruction which is executed, and the name of this instruction as well. In addition to this, at certain times, the adversary can even see the contents of all of the memory cells involved in the instruction. (This includes the instruction pointer and all of the registers which are used by the machine to determine and store the result of the computation at that time.) We will call a time when this happens a compromised time. The adversary can choose the compromised times in an adaptive way, that is, immediately before the instruction at time $t$ is executed, the adversary, using all of the information at his disposal, can decide whether time $t$ will be compromised or not. The only restriction on his choice is, that among $m$ consecutive instructions there can be at most $\varepsilon m$ whose time is compromised, where $\varepsilon>0$ is a small constant. We will call such an adversary an $(\varepsilon, m)$-moderate adversary. We show that if $m=c\lfloor\log n\rfloor$, where $c>0$ is a large constant, then for each program $P$, using $n$ memory cells and time $T=O(\operatorname{poly}(n))$, there exists an explicitly constructed and functionally equivalent program $P^{\prime}$, such that the probability that an $(\varepsilon, m)$-moderate adversary gets any nontrivial information about the input of $P$ is negligible (polynomially small in $n$ ), and the time and space requirements of $P^{\prime}$ grows, compared to $P$, only by a factor of poly $(\log n)$. We assume that the program $P^{\prime}$ gets its input in an encoded form, namely each input bit $b$ is encoded by a random 0,1 sequence of length $m$ whose parity is $b$. The output bits must be encoded by $P^{\prime}$ in a similar way. (Trivial information is the upper bound $n$ on the memory requirement of the program $P$, the upper bound $T$ on the time requirement of the program $P$ and the number and timing of the input and 
output instructions executed by $P$. We do not try to hide these values from the adversary.)

Private circuits with leaking randomly chosen wires. As part of the proof of the result about RAMs described above, we also construct, for each positive integer $m$, and for each boolean circuit $C$ of size $n$, a functionally equivalent circuit $C^{\prime}$ of size $O(n \operatorname{poly}(m))$ with the following property. The circuit $C^{\prime}$ gets each input bit $b$ of $C$ encoded as a random 0,1 sequence of length $m$ whose sum modulo 2 is $b$, and $C^{\prime}$ provides its output with the same encoding. Moreover, an adversary who can observe each bit going through the wires of the circuit $C^{\prime}$ independently with a probability of $\varepsilon>0$, where $\varepsilon$ is a small constant, can get any information about the input/output of the circuit $C$ only with a probability of at most $n e^{-c m}$, where $c>0$ is a constant. That is, if $n e^{-c m}$ is negligible, then $C^{\prime}$ is a private stateless circuit, with repsect to the adversary described above, in the sense defined in [9],

\section{THE MAIN RESULTS}

\subsection{Private circuits}

We are considering finite probabilistic boolean circuits with fan-in at most two. They may be probabilistic in the sense that in addition to their usual input nodes, that we will call deterministic input nodes, they may have probabilistic input nodes as well which get their 0,1 values at random independently and with uniform distribution. (Another essentially equivalent way to create random bits is to allow probabilistic gates, see [9].) We always assume that the set of all deterministic input nodes has a fixed ordering and make the same assumption about the output nodes as well. Assume that the probabilistic circuit $C$ has $k$ deterministic input nodes and $l$ output nodes. Then $C$ computes a random function, that is, for each $x \in\{0,1\}^{k}$, by evaluating $C$ we determine the value of a random variable $\xi_{x, C}$ which takes its values in $\{0,1\}^{l}$. The randomness of $\xi_{x, C}$ comes from the probabilistic inputs of $C$.

Suppose that $m, k, l$ are positive integers. We will say that the triplet $C=\left\langle C_{0},\left\langle I_{0}, \ldots, I_{k-1}\right\rangle,\left\langle T_{0}, \ldots, T_{l-1}\right\rangle\right\rangle$ is a block circuit with $k$ input blocks, $l$ output blocks, and blocksize $m$, if (a) $C_{0}$ is a probabilistic boolean circuit with $m k$ deterministic input nodes and $m l$ output nodes, (b) $I_{0}, \ldots, I_{k-1}$ is a partition of the set of all deterministic input nodes of $C_{0}$ into blocks of consecutive input nodes, each of size $m$, and (c) $T_{0}, \ldots, T_{l-1}$ is a partition of the set of all output nodes of $C_{0}$ into blocks of consecutive output nodes, each of size $m$. The size of $C$ is defined as the size of $C_{0}$ and it will be denoted by $|C|$.

For each $a=\left\langle a_{0}, \ldots, a_{k-1}\right\rangle \in\{0,1\}^{k}$ we define a random variable $\eta_{a, C}$ in the following way. First we select $\mathrm{km}$ random 0,1 bits $a_{i, j}, i=0,1, \ldots, k-1, j=0,1, \ldots, m-1$ independently, with uniform distribution on $\{0,1\}$ and with the condition that $\sum_{j=0}^{m-1} a_{i, j}=a_{i}$ (in $F_{2}$, the field with two elements) for $i=0,1, \ldots, m-1$. We call this step the preprocessing.

Then we place the bits $a_{i, 0}, \ldots, a_{i, m-1}$ at the input nodes in $I_{i}$ in this order, for $i=0,1, \ldots, k-1$. After that the probabilistic boolean circuit $C_{0}$ is evaluated, and we get $m l$ output bits $b_{i, j}, i=0,1, \ldots, m-1, j=0,1, \ldots, l-1$, where the bits $b_{i, j}, j=0,1, \ldots, m-1$ appear on the output nodes in $T_{i}$ in this order, for $i=0,1, \ldots, l-1$. The bits $b_{0}, \ldots, b_{l-1}$ are defined by $b_{i}=\sum_{j=0}^{m-1} b_{i, j}$, (in $F_{2}$ ). The computation of these sums is called the postprocessing. The value of the random variable $\eta_{a, C}$ is the vector $b=\left\langle b_{0}, \ldots, b_{l-1}\right\rangle$. $a=$ $\left\langle a_{0}, \ldots, a_{k-1}\right\rangle$ will be called the original input of $C$ and $b=$ $\left\langle b_{0}, \ldots, b_{l-1}\right\rangle$ will be called the original output of $C$ (referring to the motivation that the role of the block circuit $C$ will be to simulate a boolean circuit with input $a$ and output $b$.)

Assume that $C=\left\langle C_{0},\left\langle I_{0}, \ldots, I_{k-1}\right\rangle,\left\langle T_{0}, \ldots, T_{l-1}\right\rangle\right\rangle$ is a block circuit with block size $m$ and $\varepsilon>0$. We define an adversary who is observing the computation done by $C$, that is, the computation of a value of the random variable $\eta_{a, C}$ for some $a$, and tries to get some information about the pair $\langle a, b\rangle$. We will call the adversary an $\varepsilon$-random adversary if the information reaching him is determined in the following way.

Each wire of the circuit $C_{0}$ is declared compromised with a probability of $\varepsilon$ so that all of these decisions are mutually independent and also independent from the probabilistic inputs of the circuit $C_{0}$ and the randomizations during the preprocessing. The adversary gets all of the bits that are going through compromised wires during the evaluation of $C_{0}$. More precisely the adversary gets the set $W$ of all pairs $\left\langle w, b_{w}\right\rangle$ where $w$ is the name of a compromised wire and $b_{w}$ is the bit going through the wire $w$. (We assume that each wire has a unique name.) For each fixed $a \in\{0,1\}^{k}$, and $b \in\{0,1\}^{l}$, the conditional distribution of $W$ determined by the computation of the value of the random variable $\xi_{a, C}$ and the selections of the compromised wires, with the condition that $\xi_{a, C}=b$, will be denoted by $\Phi_{a, b}^{(\varepsilon, C)}$. We will say that the block circuit $C$ is $(\varepsilon, p)$-secure if, with a probability of at least $1-p$, the $\varepsilon$-random adversary does not gain any information about pair $\langle a, b\rangle$ where $a, b$ are the original input and output. That is, $C$ is $(\varepsilon, p)$-secure if for each $a \in\{0,1\}^{k}$, there exists an event $A_{a}$ with respect to all of the randomizations, such that $\operatorname{prob}\left(A_{a}\right) \geq 1-p$, and the conditional distribution of $\Phi_{a, b}^{(\varepsilon, C)}$ with the condition $A_{a}$, does not depend on the values of $a$ and $b$. (We may think that $\neg A_{a}$ is a small probability event describing a situation when we do not state anything about the knowledge of the adversary.)

Assume now that $\mathcal{C}$ is a probabilistic boolean circuit with $k$ deterministic inputs and $l$ outputs, and $C$ is a block circuit with $k$ input blocks, $l$ output blocks, and blocksize $m$. We will say that the circuit $\mathcal{C}$ and the block circuit $C$ are functionally equivalent if for each $a \in\{0,1\}^{k}$ the random variables $\xi_{a, \mathcal{C}}$ and $\eta_{a, C}$ have identical distributions.

TheOREM 1. There exist $\varepsilon>0, c>0, c_{1}>0, c_{2}>0$, such that if $m, k, l$ are positive integers, $m \geq 2$, and $\mathcal{C}$ is a probabilistic boolean circuit with $k$ deterministic input nodes and $l$ output nodes, then there exists a blockcircuit $C$ with $k$ input blocks, $l$ output blocks and with block size $m$, such that, (i) $C$ and $\mathcal{C}$ are functionally equivalent, (ii) $|C| \leq c_{1} m^{4}|\mathcal{C}|$, and (iii) $C$ is $(\varepsilon, p)$-secure, where $p=e^{-c m}|\mathcal{C}|$.

Moreover, $C$ can be constructed from $\mathcal{C}$ in time $m^{c_{2}}|\mathcal{C}|$.

Remark. 1. It is possible to show, with minor modifications of the proof, that for each fixed $\varepsilon_{1}>0$, the upper bound $|C| \leq c_{1} m^{4}|\mathcal{C}|$ can be replaced by $|C| \leq c_{1} m^{3+\varepsilon_{1}}|\mathcal{C}|$.

2 . In the proof we assume that the gates of the circuits, $\mathcal{C}$ and $C$ are performing the operations $x+y, x \times y$ and $x+1$. We prove a generalization of the theorem for an arbitrary finite field $F$, where the gates are performing these operations in the field $F$ and we also have a gate which performs the operation $-x$. In this case the inputs of the circuit $\mathcal{C}$ are not 
bits but elements of the field $F$. Each input elements $a_{i}$ is encoded by a sequence $a_{i, 0}$ from the elements of $F$ which is chosen at random and with the condition $\sum_{j=0}^{i} a_{i, j}=a_{j}$. In a similar way, the output of $C$ is an encoding of the output of $\mathcal{C}$.

\subsection{Random access machines}

We use a von Neumann type random access machine, where data and program are not distinguished. For the definition for such a machine see e.g., in [1] the random access stored program (RASP) machines, in the modified form where the contents of the memory cells are not arbitrary integers, but integers in the interval $\left[0,2^{q}-1\right]$, where $q$ is the number of bits in a single memory cell.

In order to present the main result concisely, without too many definitions, we restrict our attention to one specific RAM described in [1], with the mentioned modifications. For each positive integer $q \geq 10, \mathcal{M}_{q}$ is a machine with $2^{q}$ memory cells each containing a sequence of 0,1 bits of length $q$. These cells will be called cell $(0), \operatorname{cell}(1), \ldots, \operatorname{cell}\left(2^{q}-1\right)$. We will consider the sequences contained in the cells as the binary representations of natural numbers from the interval $\left[0,2^{q}-1\right]$, so if we say that a cell contains the natural number $i$, we mean it contains its binary representation. (We may restrict the number of memory cells if needed, by simply saying that a particular program is using only the first $n$ cells for some $n<2^{q}$. Therefore our requirement that the machine has $2^{q}-1$ memory cells, is not a real restriction). The state of the machine at each time is a function which assigns to each memory cell its content.

$\mathcal{M}_{q}$ has $\gamma_{0}$ instructions, where $\gamma_{0}$ is a constant (does not depend on $q$ ). The names or encodings of these instructions are integers in $\left[0,2^{q}-1\right]$.

$\operatorname{cell}(0)$ is called the accumulator of the machine and cell(1) the instruction pointer. (The choice of these particular cells for the mentioned roles have no significance.)

The machine $\mathcal{M}_{q}$ has six types of instructions. (a) arithmetic operations, (b) an instruction to generate a random number, (c) instructions moving data between the memory cells, (d) control transfer instructions, which determine which instruction will be executed next, (e) input/output instructions, and (f) the halt instruction to terminate the execution of the program.

The machine $\mathcal{M}_{q}$ is working in cycles, each cycle counts as one time unit. In each cycle it does the following. It checks the content of the instruction pointer. Its content is interpreted as an address, of a memory cell, say, number $i$. Then the machine executes the instruction whose name is in cell $i$. An instruction may have parameters (for the sake of simplicity we assume that each instruction has at most one parameter). A parameter typically is the address of a memory cell. The content of cell $i+1$ is considered as the parameter of the instruction in cell number $i$. The machine executes the instruction with the indicated parameter and then, if it is not a control transfer instruction, it increases the content of the instruction pointer by 2 . If it is a control transfer instruction then the instruction defines the new content of the instruction pointer. We will say that a memory cell or register is involved in an instruction if its content is used by the machine to execute the instruction, or the result of the instruction is placed in it. We will use this concept by considering an adversary who wants to get some information about what the machine is doing, and at each instruction knows which memory cells/registers are involved in the instructions, and for some of the instructions he may even know their contents.

(a) The arithmetic instructions are,$+ \times,-,\lfloor x / y\rfloor$, the constants 0,1 , and $2^{q}-1$. In case of the arithmetic operations of the form $f(x, y)$, at the time when the machine reads the instruction, which is in $\operatorname{cell}(i), x$ must be in the accumulator, and $y$ must be in the memory cell whose address is the parameter of the instruction, that is, $y$ is in $\operatorname{cell}(a)$ where $a$ is the content of $\operatorname{cell}(i+1)$. The result appears in the accumulator. In the case of the constants, the result of the instruction, that is, the constant, appears in the accumulator (and the value of the parameter is irrelevant).

(b) an instruction to generate a random number. A random integer from the interval $\left[0,2^{q}-1\right]$ appears in the accumulator, the value of the parameter is irrelevant.

(c) instructions moving data between the memory cells. Read instruction: if the value of the parameter is $a$, then the instruction puts the content of $\operatorname{cell}(a)$ into the accumulator. Write instruction: if the value of the parameter is $a$, then the instruction puts the content of the accumulator into cell $(a)$.

(d) control transfer instructions, GOTO $X$ instruction. If the value of the parameter is $X$ then the content of the instruction pointer is changed into $a$. "IF $X=0$ THEN GOTO $Y$ " instruction. If the content of the accumulator is 0 then the content of the instruction pointer is changed into $Y$, where $Y$ is the value of the parameter, otherwise the value of the instruction pointer is increased by 2 . "IF $X>0$ THEN GOTO $Y$ "If the content of the accumulator is greater than 0 , then the content of the instruction pointer is changed into $Y$, where $Y$ is the value of the parameter, otherwise the value of the instruction pointer is increased by 2 .

(e) input/output instructions. INPUT instruction. The input is written in the accumulator. OUTPUT instruction. The value of the accumulator is given as output. In both cases the value of the parameter is irrelevant.

(f) HALT instruction. Terminates the execution of the program.

The first few memory cells sometimes will be also called registers. (Intuitively this corresponds to the CPU of a computer.) A state of the machine $\mathcal{M}_{q}$, as we have indicated earlier, is a function which assigns to each of it cells a possible content.

Before the machine starts to work, a program $P_{0}$ of constant length is placed in the memory. We will call this the starting program. We may think that this is a small program, whose role is to write in the memory a larger program $P$ and data for $P$. When we will consider an adversary who wants to get some information about what the machine is doing, we will assume that $P_{0}$ is known to the adversary. Because of this, the exact way as $P_{0}$ gets into the machine is irrelevant. We usually will assume that the starting program knows what is the number of memory cells that the program can use and what is the total amount of time that can be used by the program. We assume that these two parameters are placed in the memory of the machine right after the starting program. Since we suppose that these parameters are known to the adversary, it is irrelevant what is the mechanism of placing these parameters into the machine.

We assume that a program $P$ can run on the machine in the following way. Program $P_{0}$, the starting program, is already in the machine and asks for inputs. The first part 
of the input received by $P_{0}$ is the program $P$, (when we say program $P$, some data for the program may be included in it). $P_{0}$ writes the program $P$ into the memory and then transfers the control to program $P$. While $P$ is running it may ask for input and may also provide output. In this situation we will say that the program $P_{0}$ runs the program $P$.

We will call the machine $\mathcal{M}_{q}$ described above the basic RAM.

In the definition of adversaries we will also assume that there is a limit $n$ on the number of memory cells used by the machine and a limit $T$ on the time, so that the program must stop before time $T$. These will be always known to the adversary at the time when the machine starts working. $n$ and $T$ will be also known by the starting program of $\mathcal{M}_{q}$.

The benign adversary of $\mathcal{M}_{q}$. We define an adversary, called benign adversary, for $\mathcal{M}_{q}$ who gets only information that is revealed by the actions connecting $\mathcal{M}_{q}$ to its environment, that is, information that could not be hidden anyway efficiently. Other adversaries on various type of machines will be compared to this benign adversary. Our goal will be to transform the machine $\mathcal{M}_{q}$ and its starting program in a way that an $(\varepsilon, m)$-moderate adversary with the new machine/program does not get more information about the input than the benign adversary with the same input with the original machine/program.

Definition Assume that $\mathcal{M}_{q}$ is the basic RAM, an adversary $\mathcal{B}_{q}$ is observing it, and gets the following information. (i) the upper bound $n$ on the number of memory cells used, and the upper bound $T$ on the total time used by the machine, (ii) the time of all input instructions, (iii) the time of all output instructions, (iv) the time of the $H A L T$ instruction. We also assume that $\mathcal{B}_{q}$ gets the values $n, T$ before the machine starts to work and gets each other piece of information at the time when the corresponding instruction is executed. $\mathcal{B}_{q}$ will be called the benign adversary of $\mathcal{M}_{q}$.

RAM with parity encoded i/o. In the following we describe a computational model which is the extension of the basic RAM $\mathcal{M}_{q}$, with preprocessing of the input and postprocessing of the output. Every input bit and output bit $b$ will be replaced by a random 0,1 -sequence whose sum is congruent to $b$ modulo 2 . (As we pointed out earlier without these steps an $(\varepsilon, m)$-moderate adversary could get, with a nonnegligible probability, some nontrivial information about the input.)

Definition. 1. Assume that $q \geq 10$ and $m$ are positive integers. We define a machine $\mathcal{P}_{q, m}$ in the following way. The machine gets its input as a finite sequence of integers $a_{0}, a_{1}, \ldots, a_{l-1}$ form the interval $\left[0,2^{q}-1\right]$. It will be important later that the possible inputs of $\mathcal{M}_{q}$ and $\mathcal{P}_{q}$ are the same. First the input $a_{0}, \ldots, a_{l-1}$ is transformed into a 0,1 sequence $b_{0}, b_{1}, \ldots, b_{q l-1}$ by replacing each integer with its binary representation containing exactly $q$ bits. Then each bit $b_{i}$ is replaced by a 0,1 -sequence $B_{i}=\left\langle b_{i, 0}, \ldots, b_{i, m-1}\right\rangle$ of length $m$ so that $\sum_{j=0}^{m-1} b_{i, j} \equiv b_{i}(\bmod 2)$ and the various sequences $B_{i}, i=0,1, \ldots, q l-1$ are chosen independently and with uniform distribution from the set of sequences satisfying the described condition.

Let $s=\left\langle s_{0}, s_{1}, \ldots, s_{m q l-1}\right\rangle$ be the 0,1 -sequence which is the concatenation of the sequences $B_{0}, B_{1}, \ldots$ in this order. The sequence $s$ is given to the machine $\mathcal{M}_{q}$ as an input sequence. Here we consider each $s_{i}$ as a integer in $\left[0,2^{q-1}\right]$, therefore each input instruction the machine $\mathcal{M}_{q}$ puts a sin- gle $s_{i}$, into $\operatorname{cell}(0)$. Assume that the output of the machine $\mathcal{M}_{q}$ at the input $s$ is the sequence $u_{0}, u_{1}, \ldots, u_{k-1}$, let $v_{i}=\sum_{j=i m}^{(i+1) m-1} u_{i}$, for $i=0,1, \ldots, r-1$, where $r=\lfloor k / q\rfloor-1$ and let $w_{i}$ be the least nonnegative residue of $v_{i}$ modulo 2 . The output of the machine $\mathcal{P}_{q, m}$ is the sequence $w_{0}, \ldots, w_{r-1}$.

It is irrelevant how the machine $\mathcal{P}_{q, m}$ is executing the described preprocessing and postprocessing steps since we will consider adversaries who can get only information about the various states of the machine $\mathcal{M}_{q}$ used in $\mathcal{P}_{q, m}$ but not about the preprocessing and postprocessing steps.

2. If $\mu, \nu$ are probability measures on the same $\sigma$-algebra $\mathcal{A}$, then the distance of $\mu$ and $\nu$ is $\sup \{|\mu(B)-\nu(B)|+$ $|\mu(D)-\nu(D)|\}$ taken for all $B, D \in \mathcal{A}$ with $B \cap D=\emptyset$.

3. We will denote the machine $\mathcal{M}_{q}$ by $\mathcal{M}_{q}[P, n, T]$, if it is working with the starting program $P$, with only the first $n$ memory cells, and with an upper bound $T$ on the time used. In a similar way the machine $\mathcal{P}_{q, m}$ will be denoted by $\mathcal{P}_{q, m}\left[Q, n^{\prime}, T^{\prime}\right]$ if it is using the machine $\mathcal{M}_{q}$ with the starting program $Q$, with the first $n^{\prime}$ memory cells only and with an upper bound $T^{\prime}$ on the time used by $\mathcal{M}_{q}$. We will say that the machines $\mathcal{M}_{q}[P, n, T]$ and $\mathcal{P}_{q, m}\left[Q, n^{\prime}, T^{\prime}\right]$ are functionally equivalent if for the same input sequence the distribution of their output sequences are identical. The two machines are functionally equivalent with an error of $\delta$ if the distance of the two mentioned distributions is at most $\delta$. (These distributions are determined by the random steps of $\mathcal{M}_{q}$ in both machines and by the random preprocessing of the input in $\mathcal{P}_{q, m}$.)

The $(\varepsilon, m)$-moderate adversary for $\mathcal{P}_{q, m}$. We define a set of adversaries for $\mathcal{P}_{q, m}$ that we will call the $(\varepsilon, m)$ moderate adversaries. Such an adversary will get some information about the working of the machine $\mathcal{P}_{q, m}$ at each time $t$, where we count the time $t$ in the machine $\mathcal{M}_{q}$ which works in $\mathcal{P}_{q, m}$. The information received by the adversary depends on the state of the machine $\mathcal{M}_{q}$ at time $t$ and a decision of the adversary. Namely the adversary $\mathbf{B}=\mathbf{B}_{q, m, \varepsilon}$ may declare time $t$ as a compromised time or a noncompromised time. This is done by the adversary, right before the instructions at time $t$ is executed, using all the knowledge available for him at that time. We describe below the information received by $\mathbf{B}$ at compromised or not compromised times. In the decision whether $t$ will be a compromised time $\mathbf{B}$ can use probabilistic steps. There is no computational restriction on the decision process of $\mathbf{B}$. In other words $\mathbf{B}$ has a strategy of the following form. For each possible time $t$ and for each finite 0,1 sequence $s$ (representing the information that $\mathbf{B}$ received before time $t$ ) a random variable $\xi_{t, s}$ with 0,1 values is fixed. Assume that the information that $\mathbf{B}$ got before time $t$ is represented by a 0,1 -sequence $s_{t}$. Then at time $t$ adversary $\mathbf{B}$ takes a random value of the random variables $\xi_{t, s_{t}}$ and declares the time $t$ compromised if this value is 1 . Otherwise $t$ is declared a noncompromised time.

The strategy $\mathcal{S}$ described by the random variables $\xi_{t, s}$ will be called an $(\varepsilon, m)$-moderate strategy if each time interval $I$ of length $m$ contains, with probability 1 , at most $\varepsilon m$ compromised times. An $(\varepsilon, m)$-moderate adversary is an adversary who is using an $(\varepsilon, m)$-moderate strategy for the selection of compromised times.

Assume first that $t$ is a noncompromised time. Then $\mathbf{B}$ gets the following information.

(a) the information that the benign adversary would get till time $t$, and the values of $m$ and $\varepsilon$. 
(b) the addresses of all of the memory cells involved in the instruction executed at time $t$ together with their roles in the instruction (but not their contents) and the name of the instruction.

Suppose now that $t$ is a compromised time. Then $\mathbf{B}$ gets the following information.

(c) all of the information that $\mathbf{B}$ would get if $t$ would be a noncompromised time

(d) the contents of all of the memory cells involved in the instruction executed at time $t$ (with the indication which contents belongs to which memory cells).

Measuring the strength of an adversary. We will measure the strength of an adversary (for $\mathcal{M}_{q}$ or $\mathcal{P}_{q, m}$ ) by its ability to guess correctly what was the input of the machine, provided the input was selected at random and with uniform distribution from a set $\{a, b\}$ of two elements, where the set $\{a, b\}$ is known to the adversary.

Assume that the information received by an adversary $\mathcal{V}$ till the machine $M$ has stopped is represented by a finite 0,1 -sequence $s$. If $a, b$ are possible inputs for the machine, then $\mathcal{V}$, using the information $s$, may try to guess whether $a$ or $b$ was the input. We will say that $\mathcal{V}$ can distinguish $a$ form $b$ with a bias greater than $\iota$ if there exists a function $S$ (depending on $a$ and $b$ ), so that if $h$ is a random element of the set $\{a, b\}$ with uniform distribution, and at the input $h$ the adversary $\mathcal{V}$ gets the information $s$ while the machine is working, then $\operatorname{prob}(S(s)=h)>\frac{1}{2}+\iota$, where the probability is taken for the randomization of $h$, the randomization of the machine, and the randomization of the strategy of the adversary together. $\operatorname{bias}_{\mathcal{V}}(a, b)$ will denote the largest realnumber $\delta$ so that for all $\iota<\delta$ the adversary $\mathcal{V}$ can distinguish $a$ from $b$ with a bias greater than $\iota$.

Definition. Assume that $P, Q$ are starting programs for $\mathcal{M}_{q}$ and $q, m, n, T, n^{\prime}, T^{\prime}$ are positive integers, $\varepsilon>0, \delta>0$. We will say that $\mathcal{P}_{q, m}\left[Q, n^{\prime}, T^{\prime}\right]$ with an $(\varepsilon, m)$-moderate adversary $\delta$-simulates $\mathcal{M}_{q}[P, n, T]$ with the benign adversary if the following conditions are satisfied:

(a) $\mathcal{M}_{q}\left[P, n^{\prime}, T^{\prime}\right]$ and $\mathcal{P}_{q, m}[Q, n, T]$ are functionally equivalent with an error of at most $\delta$,

(b) for all possible inputs $a, b$ we have $\operatorname{bias}_{\mathbf{B}_{q, m, \varepsilon}}(a, b) \leq$ $\operatorname{bias}_{\mathcal{B}_{q}}(a, b)+\delta$, where $\mathbf{B}_{q, m, \varepsilon}$ is the $(\varepsilon, m)$-moderate adversary defined for $\mathcal{P}_{q, m}\left[Q, n^{\prime}, T^{\prime}\right]$ and $\mathcal{B}_{q}$ is the benign adversary defined for $\mathcal{M}_{q}[P, n, T]$.

Theorem 2. There exist $c_{1}, c_{2}, c_{3}, c_{4}>0$, so that for each starting program $P$, there exists a starting program $Q$, such that for all sufficiently small $\varepsilon>0$, and for all positive integers $m, q, n, T$ with $q \geq 10, \log n \leq m \leq n \leq T \leq 2^{n}$, we have that $\mathcal{P}_{q, m}\left[Q, n m^{c_{1}}, \mathrm{Tm}^{c_{2}}\right]$, with an $(\varepsilon, m)$-moderate adversary, $\delta$-simulates $\mathcal{M}_{q}[P, n, T]$, with the benign adversary, where $\delta=c_{3} T \max \left(n^{-\log n}, e^{-c_{4} m}\right)$.

Remark 1. Note that the starting programs $P, Q$ do not depend on $q$. This is possible since we consider a program as a sequence of integers in the range $\left[0,2^{q}-1\right]$ and we suppose that the names of the instructions for $\mathcal{M}_{q}$ are fixed independently of $q$.

2. If $m=O(\log n)$ then the increase in the time requirement of $\mathcal{P}_{q, m}$ with respect to $\mathcal{M}_{q}$ is $O\left((\log n)^{\gamma}\right)$, where $\gamma \geq 1$ is a constant. $\gamma=\gamma_{1}+\gamma_{2}+\gamma_{3}+\gamma_{4}$ comes from four sources. $\gamma_{1}$ from the theorem about oblivious simulation, $\gamma_{2}$ from the circuit simulation of Theorem $1, \gamma_{3}$ from the simulation of the original $q$-bit arithmetic operations of $\mathcal{M}_{q}$ by bitwise boolean operations, and $\gamma_{4}$ from the remaining part of the proof. As the proofs of this paper are given, we have $\gamma_{2}=4$, but with minor modifications of the proof we can get $\gamma_{2}=3+\varepsilon_{1}$ for each fixed constant $\varepsilon_{1}>0$. We get $\gamma_{3}=2$ if we perform the arithmetic operations in the obvious way. Finally $\gamma_{4}=1$, and it comes from the transformation of the program in a way that the $(\varepsilon, m)$-moderate adversary will have essentially the same knowledge as an $\varepsilon$-random adversary, as will be described later. (As a consequence if we are concerned about only $\varepsilon$-random adversaries then $\gamma_{4}=0$.) The same upper bound holds for the increase of the space requirement of $\mathcal{P}_{q, m}$ compared to $\mathcal{M}_{q}$. There is however some possibility for improvement here, since while simulating the circuit $C$ of Theorem 1 with a RAM, we may repeatedly reuse the space which is needed for the evaluation of a subcircuit of $C$, which plays the role of a single gate of $\mathcal{C}$.

\subsection{Sketch of the proofs.}

The proof of Theorem 2 is using Theorem 1. Apart from the earlier results about oblivious simulation, the proof of Theorem 1 is the most important part of the proof of Theorem 2.

Sketch of the proof of Theorem 1. We may assume that the fan-in of each gate of the circuit $\mathcal{C}$ is at most two. We may also assume that the adversary chooses compromised gates and not compromised wires. The information that the adversary gets will be the bits that arrive as the input for the compromised gate. Each gate is declared compromised with a probability of $\varepsilon$ and the decisions made for the various gates are mutually independent. It is easy to see that it is sufficient to prove the theorem with this adversry.

We will construct the block circuit $C$ in the following way. We assume that in the circuit $\mathcal{C}$ there are only gates which correspond to the algebraic operations in $F_{2}$, that is we have gates for addition multiplication and adding 1 to the input. For each of the gate types,$+ \times$ and +1 we will construct a block circuit $C^{(X)}$ with block size $m$ (and with probabilistic input nodes as well) which performs the algebraic operation associated with the gate type $X$ on the values encoded by its input blocks and provides the result in encoded form on its only output block. For example if $X$ is the product gate then $C^{(X)}$ must be a circuit with two input blocks $I_{0}, I_{1}$ and one output block $T_{0}$ each of them of size $m$, so that if $a_{0}, \ldots, a_{m-1}$ are the input values on block $I_{0}, b_{0}, \ldots, b_{m-1}$ are the input values on block $I_{1}$, and $v_{0}, \ldots, v_{m-1}$ are the output values on block $T_{0}$, then they satisfy the equation $\sum_{i=0}^{m-1} v_{i}=\left(\sum_{i=0}^{m-1} a_{i}\right)\left(\sum_{j=0}^{m-1} b_{j}\right)$. This must hold independently of the values of the probabilistic inputs. That is, the block circuit $C^{(X)}$ must be functionally equivalent to the gate $X$.

In addition to the functional equivalence with gate $X$ we also want the circuit $C^{(X)}$ to be $\left(\varepsilon, p^{\prime}\right)$-secure with $p^{\prime}=$ $e^{-c_{0} m}$, for some constant $c_{0}>c_{1}$. We may hope that if we succeed in constructing such circuits $C^{(X)}$ for each of the three field operations,$+ \times$ and +1 then we may construct the required $(\varepsilon, p)$-secure circuit $C^{\prime}$ in the following way. We replace each gate $Y$ of type $X$ in $\mathcal{C}$, by a copy $Y^{\prime}$ of the circuit $C^{(X)}$, so that if the outputs of the gates $Y_{0}, Y_{1}$ are the inputs of $Y$ then the output blocks of the circuits $Y_{0}^{\prime}, Y_{1}^{\prime}$ are the input blocks of the circuit $Y^{\prime}$. Unfortunately such a $C$ is not necessarily $(\varepsilon, p)$-secure. E.g., if $X$ is the +1 gate, then $C^{(+1)}$ can be the circuit which adds 1 to the first input bit and leaves all of other input bits unchanged. It is easy to 
see that if $\mathcal{C}$ consists of a long sequence of +1 gates, then the corresponding circuit $C$ constructed in the way as indicated above is not $(\varepsilon, p)$ secure.

Therefore we will require a stronger property from the circuits $C^{(X)}$, where $X$ is a gate type. This stronger property must be inherited from $C^{(X)}$ to the circuit $C$, constructed from the building blocks $C^{(X)}$, as described above. For the formulation of this stronger property we need the following definition.

We suppose that the gates of the circuit $C$ are evaluated in some sequence, so a time is associated with each gate including the input nodes. Assume that a circuit has an $\varepsilon$-random adversary $\mathcal{X}$. At a certain time $t$, adversary $\mathcal{X}$ has some knowledge about what is happening in the circuit, because $\mathcal{X}$ got the values of the inputs of certain gates. If $\eta$ is a random variable (e.g., the encoded/original input/output), then the conditional distribution of $\eta$, with the condition what $\mathcal{X}$ knows about the evaluation of the circuit at time $t$, will be called the distribution of $\eta$ from the point of view of $\mathcal{X}$ at time $t$. Time 0 will be the time for a block circuit when the preprocessing has been done already, but the input bits has not been placed yet at the input nodes. Placing each input bit separately at the corresponding input node will require a time unit.

We consider now $\varepsilon$-random adversaries who also get some extra information. Assume that $\mathcal{Z}$ is an adversary of a block circuit, who may get some additional information about the encoded input. Such an adversary will be called an input fortified adversary. If at time 0 the distribution of the encoded input from the point of view of the input fortified adversary $\mathcal{Z}$ is $\mathcal{D}$, then $\mathcal{Z}$ will be also called a $\mathcal{D}$-input fortified adversary. Assume now that $Y$ is a subset of gates of the block circuit $C$ and the adversary gets as extra information the inputs and the output of all of the gates in $Y$. Such an adversary will be called the $Y$-set fortified adversary. If an adversary $\mathcal{Z}$ is $\mathcal{D}$-input fortified and $Y$-set fortified at the same time then we will say that $\mathcal{Z}$ is a $(\mathcal{D}, Y)$-fortified adversary.

As we have seen using a sequence of +1 gates, in spite of the fact that each of the corresponding block circuits is $(\varepsilon, p)$-secure the adversary slowly may gather information about the input of the first block circuit in this sequence and after a time will know that input completely. To avoid this, we will require more from the block circuits representing the individual gates. For the sake of simplicity let's first think of only a gate with one input and one output. The requirements on the block circuit simulating the gate will be that (i) if the knowledge of the adversary about the $m$-bit input of the block circuit has a property $P$ (that we will define later) then the knowledge the adversary about the $m$-bit output of the block circuit also have property $P$, and (ii) at the beginning of the evaluation of the block circuit the adversary has as much information about the original input (the $F_{2}$-sum of the input bits) than at the end. These properties will make it possible to connect a sequence of gates such that properties $P$ and (ii) are inherited from each circuit to the next.

Property $P$ of the distribution of the input from the point of view of the adversary will be essentially the requirement that all of the knowledge of the adversary about the input is concentrated to a small subset of input bits. More precisely property $P$ holds if it is possible to give some additional knowledge to the adversary so that he can express his total knowledge in the following way: I know a subset $Y$ of the set of input nodes and an $F_{2}$-valued function $f$ defined on $Y$, and a $\delta \in[0,1]$ such that (a) $|Y| \leq \varepsilon_{0} m$, (b) for each $y \in Y$, the input bit at the node $y \in \bar{Y}$ is $f(y)$, and (c) if $a_{1}, \ldots, a_{m-1}$, is a possible input sequence which is consistent to $f$, then the probability (from my point of view) of the event that " $a_{0}, \ldots, a_{m-1}$ is the actual input sequence" is $\delta$, if $\sum_{i=0}^{m-1} a_{i}=0$, and it is $1-\delta$ otherwise.

The value of the constant $\varepsilon_{0}$ will be chosen later. Since we consider circuits with several input blocks $I_{0}, \ldots, I_{k-1}$ the condition $|Y| \leq \varepsilon_{0} m$ will be replaced by " $\left|Y \cap I_{i}\right| \leq \varepsilon m$ for $i=0,1, \ldots, m-1$ ". This motivates the following definition of an $\varepsilon$-cylindrical distribution which corresponds to property $P$ with $\varepsilon_{0}:=\varepsilon$. The sets $W_{0}, \ldots, W_{s-1}$ are playing the role of the input blocks (or output blocks). $H$ plays the role of the fortified distribution of the input bits (or output bits) from the point of view of the adversary.

Definition 1. func $(A, B)$ will denote the set of all functions defined on $A$ with values in $B$.

2. Let $W=\left\langle W_{0}, \ldots, W_{s-1}\right\rangle$ be a finite sequence of pairwise disjoint sets, each containing exactly $m$ elements, and let $H$ be a probability distribution on func $(\bar{W}, F)$, where $\bar{W}=$ $\bigcup_{i=0}^{s-1} W_{i}, F=F_{2}$. We will say that $H$ is $\varepsilon$-cylindrical with respect to $W$, if there exists an $Y \subseteq \bar{W}$ and a function $f \in \operatorname{func}(Y, F)$ with the following properties:

(a) $\left|Y \cap W_{i}\right| \leq \varepsilon m$ for all $i=0,1, \ldots, s-1$,

(b) Assume that $a \in \operatorname{func}(\bar{W}, F)$, and $H(\{a\})>0$. Then for all $x \in Y$, we have $a(x)=f(x)$.

(c) Assume that $a, b \in \operatorname{func}(\bar{W}, F)$, and for all $i=$ $0,1, \ldots, s-1, \sum_{x \in W_{i}} a(x)=\sum_{x \in W_{i}} b(x)$, and for all $x \in Y$, $a(x)=b(x)=f(x)$. Then $H(\{a\})=H(\{b\})$. (That is, if an $a \in \operatorname{func}(\bar{W}, F)$ is an extension of $f$, then $H(a)$ depends only on the sequence $\sum_{x \in W_{i}} a(x), i=0, \ldots, s-1$.)

A function $f$ with the described properties will be called a handle of the distribution $H$, and the corresponding set $Y=\operatorname{domain}(f)$ will be called a base set of $H$.

In other words, a probability distribution $H$ on func $(\bar{W}, F)$ is $\varepsilon$-cylindrical if each function which has a positive probability has the same predetermined values on the base set $Y$, which intersects each $W_{i}$ in at most $\varepsilon m$ elements, and for all $a \in \operatorname{func}(\bar{W}, F)$ if $H(\{a\}) \neq 0$ then $H(\{a\})$ depends only on the sums $\sum_{x \in W_{i}} a(x)$ for $i=0,1, \ldots, s-1$.

Next we define the notion of an $\left(\varepsilon_{0}, \varepsilon_{1}, p\right)$-cylindrical block circuit. The parameter $\varepsilon_{1}$ refers to the fact that we consider the behavior of the circuit respect to an $\varepsilon_{1}$-random adversary. The definition, roughly speaking, expresses the following property of the circuit: if the distribution of the input is $\varepsilon_{0}$-cylindrical, from the point of view of the $\varepsilon_{1}$-random adversary, then, with a probability of at least $1-p$, the distribution of the output is also $\varepsilon_{0}$-cylindrical. That is, if all of the knowledge of the adversary about the input can be concentrated on a set of input nodes $B$ which intersects each input block in a subset with at most $\varepsilon_{0} m$ elements, then the ouput, with a probability of at least $1-p$, has the same property. We also require that the knowledge of the adversary about the original input does not increase.

Definition 1. $a \ll b_{1}, \ldots, b_{k}$ will mean: " $a$ is sufficiently small with respect to $b_{1}, \ldots, b_{k}$ ".

2. Assume that $\varepsilon_{0}, \varepsilon_{1}, p$ are reals. We will say that the block circuit $C$ with $k$ input blocks and $l$ output blocks of sizes $m$, is $\left(\varepsilon_{0}, \varepsilon_{1}, p\right)$-cylindrical if the following holds. Suppose that an input fortified $\varepsilon_{1}$-random adversary knows at 
time 0 that the encoded input is arriving with distribution $\mathcal{D}$, where $\mathcal{D}$ is an $\varepsilon_{0}$-cylindrical distribution on the encoded input, with respect to the input block sequence, and with base set $B$. Assume further that the same $\varepsilon_{1}$-random adversary selects, by the random selection process, a set $D$ of compromised gates. Then with a probability of at least $1-p$ for the randomization of the probabilistic inputs of $C$, there exists a set $\mathbf{X} \supseteq B \cup D$ of the gates of $C$ with the following properties.

(a) the distribution of the encoded output from the point of view of a $(\mathcal{D}, \mathbf{X})$-fortified $\varepsilon_{1}$-random adversary, after the computation by the circuit has been completed, is $\varepsilon_{0^{-}}$ cylindrical with base set $\mathbf{X} \cap \bigcup_{j=0}^{l-1} T_{j}$, where $T_{0}, \ldots, T_{l-1}$ are the output blocks.

(b) The distribution of the original input as determined by $\mathcal{D}$, that is, the distribution of the original input from the point of view of the $\mathcal{D}$-input fortified adversary at time 0 , is the same as the distribution of the original input from the point of view of the $(\mathcal{D}, \mathbf{X})$-fortified $\varepsilon_{1}$-random adversary (who selected the compromised set $D$ ) when the computation done by the circuit has been completed.

We will prove that if $X$ is a gate type, that is,,$+ \times$ or +1 , then for all $\varepsilon_{0}, \varepsilon_{1}, c$ with $0<\varepsilon_{1} \ll c \ll \varepsilon_{0} \ll 1$, and for all sufficiently large $m$, there exists a block circuit $C_{m}^{(X)}$ with block size $m$ so that: (1) $C_{m}^{(X)}$ is functionally equivalent to gate $X,(2) C^{(X)}$ is $\left(\varepsilon_{0}, \varepsilon_{1}, e^{-c m}\right)$-cylindrical, (3) the size of $C^{(X)}$ is polynomial in $m$ and the circuit $C^{(X)}$ can be constructed by a Turing machine in time polynomial in $m$.

Using this statement, it can be easily proved by recursion on the size of the circuits, that for all $\varepsilon_{0}, \varepsilon_{1}, c$ with $0<$ $\varepsilon_{1} \ll c \ll \varepsilon_{0} \ll 1$, and for all sufficiently large $m$, and for all circuits $\mathcal{C}$, there exists a block circuit $C$ with block size $m$ so that: (1) $C$ is functionally equivalent to $\mathcal{C}$, (2) $C$ is $\left(\varepsilon_{0}, \varepsilon_{1}, p\right)$-cylindrical, with $p=|\mathcal{C}| e^{-c m},(3)$ the size of $C$ is at most poly $(m)|\mathcal{C}|$, and the circuit $C$ can be constructed by a RAM in time poly $(m)|\mathcal{C}|$.

Before we construct the gates $C^{(X)}$ for the gates,$+ \times$ and +1 , we define a new gate, the identity gate whose input is same as its output. The corresponding block circuit with block size $m$, that we will construct, will be denoted by $\mathbf{C}_{m}^{(=)}$. We want to construct $\mathbf{C}_{m}^{(=)}$so that it has one input block, one output block, and if the encoded input is $b_{0}, \ldots, b_{m-1}$, the encoded output is $a_{0}, \ldots, a_{m-1}$, then $\sum_{i=0}^{m-1} b_{i}=\sum_{0}^{m-1} a_{i}$, and for all $0<\varepsilon_{1} \ll c_{1} \ll \varepsilon_{0} \ll 1$, and for all sufficiently large $m$ the circuit $\mathbf{C}_{m}^{(=)}$is $\left(\varepsilon_{0}, \varepsilon_{1}, e^{-c_{1} m}\right)$-cylindrical. Actually it will have a stronger property, namely in the definition of $\left(\varepsilon_{0}, \varepsilon_{1}, e^{-c_{1} m}\right)$-cylindricity we may replace the assumption that the encoded input is $\varepsilon_{0}$-cylindrical by a weaker assumption that it is $\varepsilon_{2}$-cylindrical where $\varepsilon_{0} \leq \varepsilon_{2} \ll 1$. This means that if during the construction of $\mathcal{C}$, from circuits of type $C^{(X)}$, the parameter $\varepsilon_{0}$ is degrading, gets larger because the adversary have seen too much, then we can reset it to its original value by simply inserting a $\mathbf{C}_{m}^{(=)}$circuit in the construction of $\mathcal{C}$. Since this corresponds to the identity gate it will not change the functionality of $\mathcal{C}$. We will also call the circuit $\mathbf{C}_{m}^{(=)}$the copying circuit. Its construction is based on a constant degree expander.

The circuit $C_{m}^{(+)}$will simply execute the addition separately for each component and then apply the circuit $\mathbf{C}_{m}^{(=)}$ to the output. The circuit $C_{m}^{(+1)}$ in a similar way adds 1 to the first component then applies $\mathbf{C}_{m}^{(=)}$to the output.
The most problematic part of the proof is the construction of $C^{(\times)}$, where direct computation would reveal the input for an $\varepsilon$-random adversary. We only sketch here the construction. Suppose we want to compute the product $a b$. We may split $a$ at random into a sum $a=a_{0}+a_{1}$ (In the block circuit $C^{(\times)}$this calculation and randomization will be done with sequences of length $m$ encoding $a, a_{0}, a_{1}$.) We have $a b=a_{0} b+a_{1} b$. Now we split $b$ at random into two parts but do this independently at the two occurrences of $b$. We get $a b=a_{0} b_{0}+a_{1} b_{1}+a_{1} b_{2}+a_{1} b_{3}$ where $b=b_{0}+b_{1}=b_{2}+b_{3}$. As the next step we split $a_{0}, a_{1}$ into two parts independently at their various occurrences, and get for $a b$ a sum with eight terms, each is a product where the first factor was derived from $a$ the second from $b$. We continue this, alternately splitting the first or second factors from each term at random independently and with uniform distribution. Assuming that $m=2^{d}$, after $3 d$ steps we will have a sum with $m^{3}$ terms, each one is a product. The block circuit now actually computes each of these $m^{3}$ products after adding the elements of the sequences of length $m$. It is important that the addition is done on a binary tree of depth $d$ whose nodes are + gates. After both factors are computed of a term, they are multiplied and the block circuit has the value of the term in an unencoded form. Of course the $\varepsilon_{1}$-random adversary may see some of these products. Now we encode, by a random 0,1 -sequence of length $m$, each of the $m^{3}$ products. Each encoding is calculated on the nodes of a binary tree with $m$ leaves, starting at the root, getting the encoding on the leaves, and randomly splitting each bit on a node into a random sum given on its two children. Finally the block circuit adds the $\mathrm{m}^{3}$ products in their encoded forms. This is done by repeatedly using the circuit $\mathbf{C}_{m}^{(+)}$.

Sketch of the proof of Theorem 2. For the sake of simplicity we assume that $m=O(\log n)$. As a first step in the proof of Theorem 2, we show that it is enough to prove it in the special case, when we replace the $(\varepsilon, m)$-moderate adversary with another $\varepsilon$-random adversary. We will call an adversary for the machine $\mathcal{P}_{q, m}$ an $\varepsilon$-random adversary, if the compromised times are selected by the adversary at random in a way that for each fixed $t$ the probability that $t$ is compromised is at most $\varepsilon$ and the events " $t$ is compromised" for various values of $t$ are mutually independent. An $\varepsilon$-random adversary gets the same information as the $(\varepsilon, m)$-moderate adversary would get with the same choices of compromised time.

Assume that the theorem holds for $c \varepsilon$-random adversaries, for some constant $c>1$, that we will fix later. Let $Q^{\prime}$ be the starting program of $\mathcal{P}_{m, q}$ such that $\mathcal{P}_{m, q}\left[Q^{\prime}\right]$ is secure against $c \varepsilon$-random adversaries. We want to define the starting program $Q$ so that $\mathcal{M}_{m, q}[Q]$ is secure against $(\varepsilon, m)$ moderate adversaries. We define $Q$ in a way that $\mathcal{M}_{q}[Q]$ simulates the machine $\mathcal{M}_{q}\left[Q^{\prime}\right]$, and so $\mathcal{P}_{q, m}[Q]$ simulates the machine $\mathcal{P}_{q, m}\left[Q^{\prime}\right]$. The simulation will simply delay each step of $\mathcal{M}_{q}\left[Q^{\prime}\right]$ by a random amount of time. More precisely before $Q$ executes instruction $X$ in this simulation, $Q$ generates a random integer $g \in[0, m-1]$ with uniform distribution, and if $g=0$, then $Q$ executes $X$ in the simulation of $\mathcal{M}_{q}\left[Q^{\prime}\right]$ by using no more than $c$ time units for some constant $c>0$. Otherwise, instead of executing $X$, it generates another random integer $g$ etc., till it gets a $g=0$ and instruction $X$ is executed. (The constant $c$ may be greater than 1 since the simulation of a single instruction in a controlled way requires several instructions.) For such a $Q$ 
we can easily define a $c \varepsilon$-random adversary $\mathcal{X}$ who knows at least as much as the $(\varepsilon, m)$-moderate adversary $\mathbf{B}$. Since the theorem holds for $\mathcal{X}$ it has to hold for $\mathbf{B}$ as well.

For the proof of Theorem 2 with an $\varepsilon$-random adversary we use the results of [3] and [2] about oblivious simulation. It is proved there that every RAM $M_{1}$ can be obliviously simulated by another RAM $M_{2}$ of the following structure. The memory cells of the simulating RAM are partitioned into two sets. The first set contains $\operatorname{cell}(0), \operatorname{cell}(1), \ldots$, $\operatorname{cell}(c)$ (including the instruction pointer and the accumulator) where $c$ is a constant. This will be called the CPU. The set of remaining cells is called the memory. A program is running in the CPU, and the memory is used only for storing and retrieving individual bits, that is, the content of each memory cell $\operatorname{cell}(k+1), \operatorname{cell}(k+2), \ldots$ is always 0 or $1 . M_{1}$ and $M_{2}$ are functionally equivalent, the time and space requirements of $M_{2}$ is increased, compared to $M_{1}$ only by a poly $(\log (n))$ factor, and an adversary who knows at each time which instruction is executed in $M_{2}$ and what are the addresses of the memory cells involved in the instruction, with high probability does not gain any nontrivial information about the input of the machine. It is an easy consequence of this fact that the simulation of $M_{1}$ can also be done by a different type of machine that we will call a composite machine, which have the additional nice property that the adversary may also see the contents of each memory cells in the CPU (but not in the memory) at each time. We only give here the definition of the machine, the proof is based on the idea that each state of the CPU of $M_{2}$ can be represented in the memory and the change from such a represented state to the next one can be performed by a sequence of boolean operations on the individual bits of this representation. Moreover the sequence of these boolean operation is given once and for all, it does not depend on the input of the machine.

The Composite machine. The composite machine, that will be denoted by $\mathcal{C}_{q, c, k}$, will depend on three integer parameters $q, c$ and $k, q=O(\log n)), k=O(n$ poly $(\log n))$. The machine $\mathcal{C}_{q, c, k}$ has two parts. The first part will be denoted by $\overline{\mathcal{M}}_{q, c}$. This will work as a $\mathrm{RAM} \mathcal{M}_{q, c}$, (that is, a RAM with word length $q$ and with $c$ memory cells), but with a special interpretation on its input and output instructions. Namely the output and input instructions will be used only for communication between $\overline{\mathcal{M}}_{q, c}$ and the second part of the composite machine which will be denoted by $\mathcal{A}_{k} . \mathcal{A}_{k}$ has $k$ memory cells each containing 1 bit. We may think of $\mathcal{C}_{q, c, k}$ as a $R A M \mathcal{M}_{q, c}$ with only $c$ memory cells, but with a large external memory $\mathcal{A}_{k}$ containing $k$ bits. $\mathcal{M}_{q, c}$ through its output instruction is able to perform boolean operations on the bits stored in $\mathcal{A}_{k}$, and also to move individual bits inside $\mathcal{A}_{k} . \mathcal{M}_{q, c}$ with its input instruction is able to read individual bits stored in $\mathcal{A}_{k}$.

Definition. We define a machine $\mathcal{A}_{k}$ with $k$ memory cells $x_{0}, \ldots, x_{k-1}$ each containing a single bit of information, 0 or 1. The machine will be called a semi-RAM because it will have instructions, but will not have a mechanism to decide which is the next instruction to be executed. Such a decision will come always from an outside source. The machine $\mathcal{A}_{k}$ has the instructions listed below. After the name of the instruction we describe its effect. (The last instruction does not change the content of any of the memory cells, yet it will have a useful role when we will simulate $\mathcal{A}_{k}$ with another machine.)
INPUT. The next bit of information from an input buffer, is written into the memory cell $x_{0}$.

OUTPUT. The content of memory cell $x_{0}$ is given as output.

WRITE $i$. Assume that $A$ is the content of cell $x_{0}$. The content of cell $x_{i}$ is changed into $A$.

READ $i$. Assume that $A$ is the content of cell $x_{i}$. The content of cell $x_{0}$ is changed into $A$.

NEGATION. The content of cell $x_{0}$ is replaced by its boolean negation.

AND. Assume that $A_{i}$ is the content of cell $x_{i}$ for $i=0,1$. The content of cell $x_{0}$ is replaced by $A_{0} \wedge A_{1}$.

EXCLUSIVE OR. Assume that $A_{i}$ is the content of cell $x_{i}$ for $i=0,1$. The content of cell $x_{0}$ is replaced by $A_{0}+A_{1}$ where + denotes the boolean operation "exclusive or" (or equivalently addition in the field $F_{2}$ ).

RANDOM. A random 0,1 bit with uniform distribution is written into cell $x_{0}$.

REFRESH. There is no change in the contents of the memory cells.

Remark 1. The machine $\mathcal{A}_{k}$ does not have a way to decide which instruction will be executed. We will define another machine, machine $\overline{\mathcal{M}}_{q, c}$ below which will make this decision.

2. The INPUT and OUTPUT instructions of $\mathcal{A}_{k}$ communicate with the outside world and not with the machine $\overline{\mathcal{M}}_{q, c}$.

Definition We define a machine $\mathcal{C}=\mathcal{C}_{q, c, k}=\left\langle\mathcal{A}_{k}, \overline{\mathcal{M}}_{q, c}\right\rangle$ which consists of two parts: a semi-RAM $\mathcal{A}_{k}$ and a RAM $\overline{\mathcal{M}}_{q, c}$ which is a machine of the type $\mathcal{M}_{q}$ with exactly $c$ memory cells, but its input and output instruction work in a different way than in $\mathcal{M}_{q}$. The machine $\mathcal{C}$ will be called a composite-RAM. $\mathcal{C}$ works in the following way. We assume that each of the instruction of $\mathcal{A}_{k}$ has a name represented by a natural number which can be the content of a memory cell of $\overline{\mathcal{M}}_{q, c}$, and so can be an output of $\overline{\mathcal{M}}_{q, c}$. When the machine starts there is a deterministic program $P_{0}$ already in the memory of $\overline{\mathcal{M}}_{q, c}$, which immediately starts to work. Suppose that $\overline{\mathcal{M}}_{q, c}$ gives an output which is the name of an $\mathcal{A}_{k}$ instruction, possibly together with a parameter. Then $\mathcal{A}_{k}$ immediately executes this instruction, and then $\overline{\mathcal{M}}_{q, c}$ continues its work. When $\overline{\mathcal{M}}_{q, c}$ executes an input instruction then the content of the memory cell $x_{0}$ of $\mathcal{A}_{k}$ is written immediately into cell(0) of $\overline{\mathcal{M}}_{q, c}$ and $\mathcal{A}_{k}$ executes a REFRESH instruction. The composite machine $\mathcal{C}_{q, c, k}=\left\langle\overline{\mathcal{M}}_{q, c}, \mathcal{A}_{k}\right\rangle$ works this way till $\overline{\mathcal{M}}_{q, c}$ executes HALT instruction when the machine $\mathcal{C}$ stops.

Remark 1. The memory cells of $\overline{\mathcal{M}}_{q, c}$ and $\mathcal{A}_{k}$ are completely separate. There is no other information transfer between the two machine, than the ones explicitly described in the definition of $\mathcal{C}$. E.g., the outputs of $\mathcal{A}_{k}$ will not necessarily reach $\overline{\mathcal{M}}_{q, c}$, we may think of them as outputs to the outside word. Of course if $\overline{\mathcal{M}}_{q, c}$ needs this information it can get it using its own input instruction which copies the content of $x_{0}$ into cell(0).

2. When $\overline{\mathcal{M}}_{q, c}$ gives an output which is a name of a $\mathcal{A}_{k}$ instruction then, as we said in the definition, the corresponding instruction is executed in $\mathcal{A}_{k}$, however this name (or its parameter) is not considered as an input for $\mathcal{A}_{k}$.

3. There is no instruction of $\overline{\mathcal{M}}_{q, c}$ which writes a 0,1 -bit into a memory cell of $\mathcal{A}_{k}$. This however can be accomplished using other instructions. For example, the instruction sequence, "WRITE 1, NEGATION, AND" puts a 0 into $x_{0}$. 
The results of [3] and [2] about oblivious simulation has the following easy consequence that we formulate in terms of composite machines. For the sketch of this proof we give only an informal statement.

Lemma A. (Informal statement.) For each $R A M M_{1}$, with $n$ memory cells each containing $q=O(\log n)$ bits, there exists a composite machine $\mathcal{C}=\mathcal{C}_{q, c, k}$ with $k=$ $O(n$ poly $(\log n))$ which is functionally equivalent to $M_{1}$ (assuming here that $M_{1}$ gets its input and provide its output bit by bit) such that the following holds. Suppose that $\mathcal{X}$ is an adversary who knows at each time the contents of all of the memory cells in $\overline{\mathcal{M}}_{q, c}$ and (as a consequence) which instruction is executed in the machines $\overline{\mathcal{M}}_{q, c}, \mathcal{A}_{k}$, and which are the addresses of the memory cells involved in these instructions, (but $\mathcal{X}$ does not get directly any information about the contents of the memory cells of $\mathcal{A}_{k}$ ). Then $\mathcal{X}$ with high probability will not get any nontrivial information about the input of the machine $M_{1}$.

The next step in the proof of Theorem 2 is that using Theorem 1 we simulate the composite machine $\mathcal{C}_{c, q, k}$ on a RAM $\mathcal{M}^{\prime}=\mathcal{P}_{q, m}$, with $n^{\prime}$ memory cells, where $n^{\prime}=$ $O(k \operatorname{poly}(\log n))$, which gets the input bits in a parity encoded form with encoding sequences of length $m=O(\log n)$. In this simulation we represent each bit $b$ contained in a memory cell $x_{i}$ of $\mathcal{A}_{k}$ by a 0,1 sequence of length $m$ whose parity is $b$, and whose elements are stored in separate memory cells. $\mathcal{M}^{\prime}$ preforms each instruction of $\overline{\mathcal{M}}_{q, c}$, with the exception of output and input instructions in their original forms which will not cause any problem since the adversary will know the contents of the memory cells in $\overline{\mathcal{M}}_{q, c}$ anyhow. At an output instruction of $\overline{\mathcal{M}}_{q, c}$, the machine $\mathcal{M}^{\prime}$ performs the corresponding $\mathcal{A}_{k}$ instruction using the $m$-bit representations of the contents of the memory cells of $\mathcal{A}_{k}$. In the case of the instructions NEGATION, AND and "EXCLUSIVE OR", this step is done by $\mathcal{M}^{\prime}$ using the block circuits, simulated by the RAM, whose existence is guaranteed by Theorem 1. For instructions WRITE, READ, and REFRESH the circuit $\mathbf{C}_{m}^{(=)}$is used. The random instruction is simply the bit by bit randomization of a 0,1 sequence of length $m$. The INPUT instruction of $\mathcal{A}_{k}$ is simulated by $\mathcal{M}^{\prime}$ by making a 0,1 -sequence of length $m$ from the next $m$ input bits of $\mathcal{M}^{\prime}$. The output instruction of $\mathcal{A}_{k}$ is simulated by $\mathcal{M}^{\prime}$ by giving the bits of the sequence, representing the content of cell $x_{0}$, as an output.

Finally when $\overline{\mathcal{M}}_{q, c}$ executes an INPUT instruction then $\mathcal{M}^{\prime}$ adds the bits of the sequence representing the content of $x_{0}$ and writes it into cell(0) of $\overline{\mathcal{M}}_{q, c}$ and then simulates the REFRESH instruction the same way as described above.

The definition of $\mathcal{M}^{\prime}$ implies that it is functionally equivalent to $M_{1}$. As we have indicated already, we apply Theorem 1 to the circuits simulated by $\mathcal{M}^{\prime}$. Using this and Lemma $\mathbf{A}$, we can prove that an $\varepsilon$-random adversary of $\mathcal{M}^{\prime}$, with high probability does not get any nontrivial information about the input of $M_{1}$.

\section{REFERENCES}

[1] A. V. Aho, J. E. Hopcroft, and J. D. Ullman. The Design and Analysis of Computer Algorithms. Addison-Wesley Publishing Company, Reading, Massachusetts, 1974.

[2] M. Ajtai. Oblivious $R A M$ s without cryptographic assumptions. Electronic Colloquium on Computational Complexity (ECCC), 17:28, 2010.

[3] M. Ajtai. Oblivious $R A M$ s without cryptogrpahic assumptions. In L. J. Schulman, editor, Proceedings of the 42nd ACM Symposium on Theory of Computing, STOC 2010, Cambridge, Massachusetts, USA, 5-8 June 2010, pages 181-190. ACM, 2010.

[4] I. Damgård, S. Meldgaard, and J. B. Nielsen. Perfectly secure oblivious $R A M$ without random oracles. Cryptology ePrint Archive, Report 2010/108, 2010. To appear in TCC 2011.

[5] S. Faust, T. Rabin, L. Reyzin, E. Tromer, and V. Vaikuntanathan. Protecting circuits from leakage: the computationally-bounded and noisy cases. In H. Gilbert, editor, Advances in Cryptology EUROCRYPT 2010, 29th Annual International Conference on the Theory and Applications of Cryptographic Techniques, French Riviera, May 30 June 3, 2010. Proceedings, volume 6110 of Lecture Notes in Computer Science, pages 135-156. Springer, 2010.

[6] O. Goldreich. Towards a theory of software protection and simulation by oblivious $R A M \mathrm{~s}$. In $S T O C$, Proceedings of the Nineteenth Annual ACM Symposium on Theory of Computing, 25-27 May 1987, New York City, NY, USA, pages 182-194. ACM, 1987.

[7] O. Goldreich and R. Ostrovsky. Software protection and simulation on oblivious $R A M \mathrm{~s}$. J. ACM, 43(3):431-473, 1996.

[8] S. Goldwasser and G. N. Rothblum. Securing computation against continuous leakage. In T. Rabin, editor, Advances in Cryptology - CRYPTO 2010, 30th Annual Cryptology Conference, Santa Barbara, CA, USA, August 15-19, 2010. Proceedings, volume 6223 of Lecture Notes in Computer Science, pages 59-79. Springer, 2010.

[9] Y. Ishai, A. Sahai, and D. Wagner. Private circuits: Securing hardware against probing attacks. In D. Boneh, editor, Advances in Cryptology - CRYPTO 2003, 23rd Annual International Cryptology Conference, Santa Barbara, California, USA, August 17-21, 2003, Proceedings, volume 2729 of Lecture Notes in Computer Science, pages 463-481. Springer, 2003.

[10] S. Micali and L. Reyzin. Physically observable cryptography (extended abstract). In M. Naor, editor, Theory of Cryptography, First Theory of Cryptography Conference, TCC 2004, Cambridge, MA, USA, February 19-21, 2004, Proceedings, volume 2951 of Lecture Notes in Computer Science, pages 278-296. Springer, 2004.

[11] R. Ostrovsky. Efficient computation on oblivious $R A M$ s. In STOC, Proceedings of the Twenty Second Annual ACM Symposium on Theory of Computing, 14-16 May 1990, Baltimore, Maryland, USA, pages 514-523. ACM, 1990.

[12] R. Ostrovsky. Software Protection and Simulation on Oblivious RAMs. PhD thesis, MIT, 1992.

[13] N. Pippenger and M. J. Fischer. Relations among complexity measures. J. ACM, 26(2):361-381, 1979. 